\title{
Automated Hydroponics with Remote Monitoring and Control Using IoT
}

\author{
Dr. Asawari Dudwadkar ${ }^{1}$, Tarit Das ${ }^{2}$, Sakshi Suryawanshi², Rajeshwari Dolas ${ }^{2}$, Tejas Kothawade ${ }^{2}$ \\ ${ }^{1}$ Assistant Professor, ${ }^{2}$ Undergraduate Student, \\ Department of Electronics Engineering \\ V.E.S. Institute of Technology \\ Mumbai, India
}

\begin{abstract}
Today, India wants to be fitter and this healthconsciousness drives for healthier food. Vegetables available in urban India are usually highly contaminated with pesticides wrecking our health and the cultivating land instead. Organic Farming as an alternative is not very feasible due to its exorbitant prices. Hydroponics along with our designed system offers a new way of cultivation which promotes all aspects of sustainable agriculture thereby providing an alternative to organic farming. In this paper, we have designed a hydroponic system which ensures higher growth rate with controlled environment. The main function of our hydroponic system is to monitor and to control parameters with the help of actuators present in the system to produce plants and exotic vegetables at a faster rate and by revoking the necessity of the arduous activities generally associated with farming. Apart from that, remote monitoring and controlling of the artificial environment to eliminate the dependency on the natural habitat to ensure year-round availability of all plants and vegetables is one of the main objectives of this project. The project shows that hydroponic systems which are monitored and controlled properly show higher rates of growth and also reduce human dependency.
\end{abstract}

Keywords-Hydroponics, IoT, Sensors

\section{INTRODUCTION}

Hydroponics is the process of growing plants in the absence of soil with the help of added nutrients. The roots of the plants are directly exposed to the mineral nutrient solution in the water solvent [1]. This ensures the healthy growth of the plant. Apart from the nutrient solution, regulating the artificial atmospheric conditions is also an important contributing factor in ensuring the growth of the plant, which is generally $25-30 \%$ faster with higher yield [2].

Due to constraints of farming areas, farmers hugely depend on intensive farming with added amounts of fertilizers, degrading the quality of soil. Its dependency on the natural elements leaves him in a very vulnerable state to decide the quantity, quality and the type of plant to harvest.

Urban India drives the major vegetable demand of the country contributing effectively in the import of numerous exotic vegetables in high amounts. With close monitoring and the ability to modify and control the specifics of the environment in which the plant is supposed to grow, the producers can effortlessly meet the requirements of the market. Apart from the mass production, our system will also help and prove beneficial to small scale cultivators, urban producers and individuals who want to grow vegetables in their backyard.

\section{II.IDEOLOGY AND APPROACH}

The most important ideology behind this project was to collectively use the Greenhouse Principle and Principle of Nutrition Film technique in Hydroponics to maximize the growth of plants [1]. So in order to make this worth executing in our daily lives, we worked on developing a system which was

- Closed and Self Sustainable

- Where Parameters can be monitored easily

- Supported Hydroponics

All these things put together formed the physical structure of the project. But, we also needed data to make the system automatic. Raw data was collected from the system which was monitored beforehand. The data was then processed using Exploratory and Graphical data analysis to determine and form equations so that the microcontroller could predict the required values [3]. Then, with the help of the lead-lag compensating control mechanism, the external system approaches stability.

\section{III.THE ACTUAL SYSTEM}

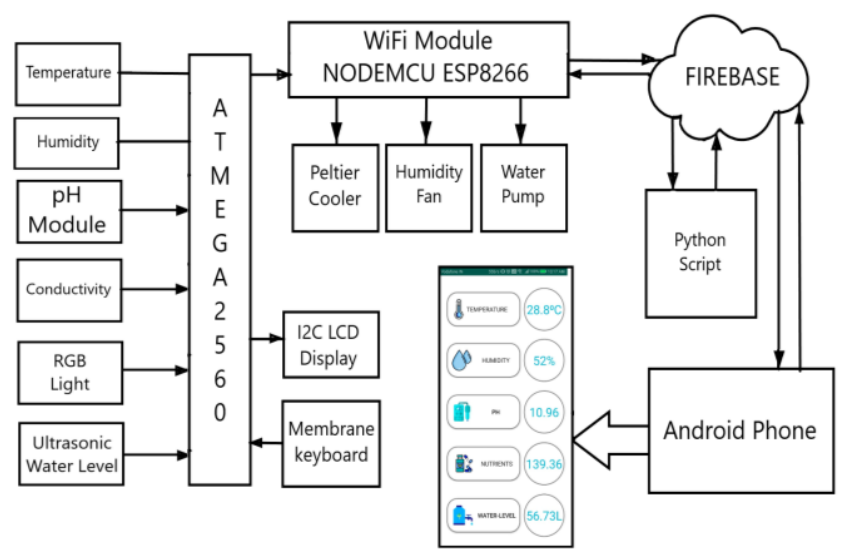

Fig. 1. Functional Block Diagram of Complete system

The main function of our hydroponic system is to monitor and try to control parameters with the help of actuators present in the system. Apart from that, Remote monitoring and controlling are one of the main objectives.

The entire system can be divided into two subsystems (Hardware and the Software). The hardware assembly is responsible for the processes and control over the system. This includes circuitry which contains sensors like temperature and humidity sensor, $\mathrm{pH}$ sensor, conductivity plate sensors, all of which will determine the condition of water and the environment where the plants are kept. Along 
with sensors, the circuitry also consists of actuators like the water pump, air pump and Peltier cooling system. All sensors and actuators are connected to the microcontroller.

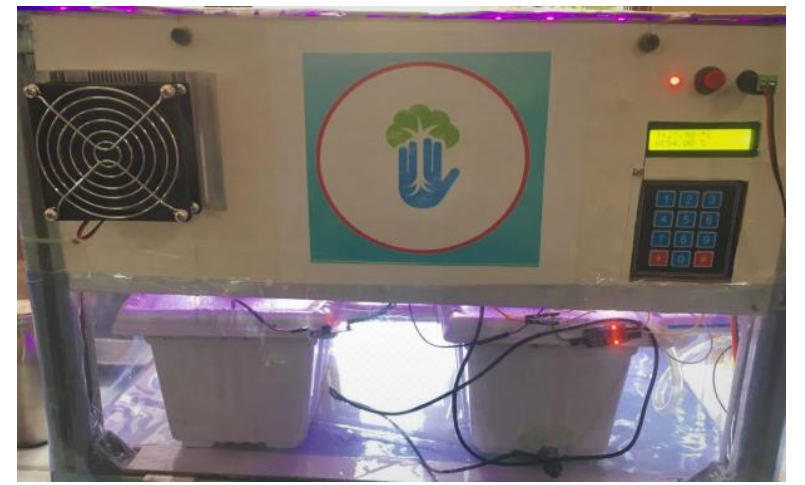

Fig. 2. Actual hardware system implemented

The microcontroller looks after the control of the system. It checks all the parameters like temperature, humidity, $\mathrm{pH}$ level, conductivity, real-time and water level in the external tank. In hydroponics, the main objective is to transfer all necessary and sufficient nutrients to plants whose roots are suspended in the water present in the system. Analyzing these conditions, we have designed some prospects that have been carried out in our system.

The software part of the system includes a networking module and a controller which is connected to the microcontroller. This networking module is also connected to an online database which updates the sensor values and status of the actuators. Because of this, the system can be monitored and controlled from a distance. All this data can be seen and controlled on our Android Application.

\section{IV.DATA ANALYSIS}

The main data was collected (118 samples) from our system and about 80 samples were used to train the model with the help of polynomial regression in python [3]. We made sets of data to train models and predict the action. Python 3 has libraries: NumPy, Pandas, which helped in analysis and creating equations for better understanding.

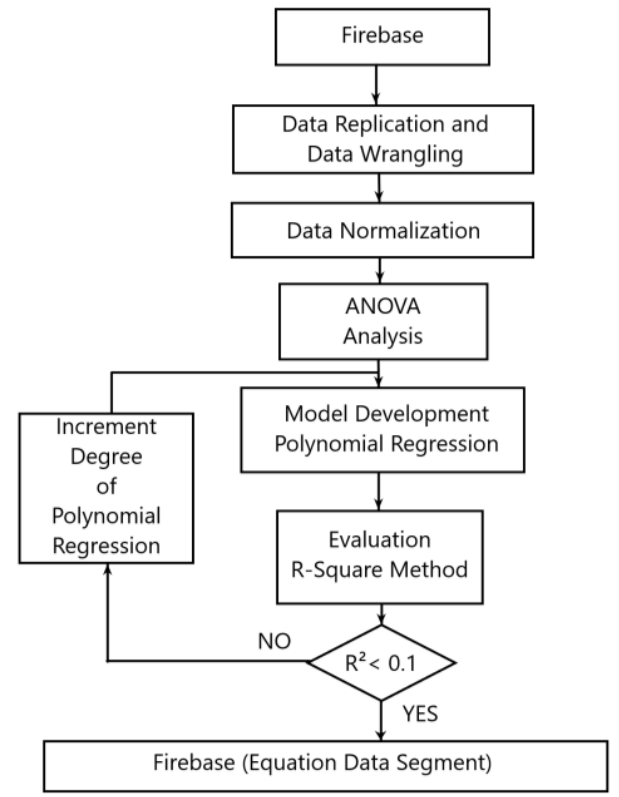

Fig. 3. Flowchart of Python Scripting
The variables we predicted were the quantity of nutrient fluid to be added in the water. The independent variables we had were $\mathrm{pH}$ level, conductivity percentage, temperature, humidity and the water level of the tank.

After individual analysis of every parameter, the graphical analysis particularly shows that the external surroundings help in the growth of the plant, and the change in nutrient requirements is slightly observed.

On looking at the graphs, Humidity may not seem a factor. But on observing the dataset, we find that the variation in the temperature graph is due to change in humidity.

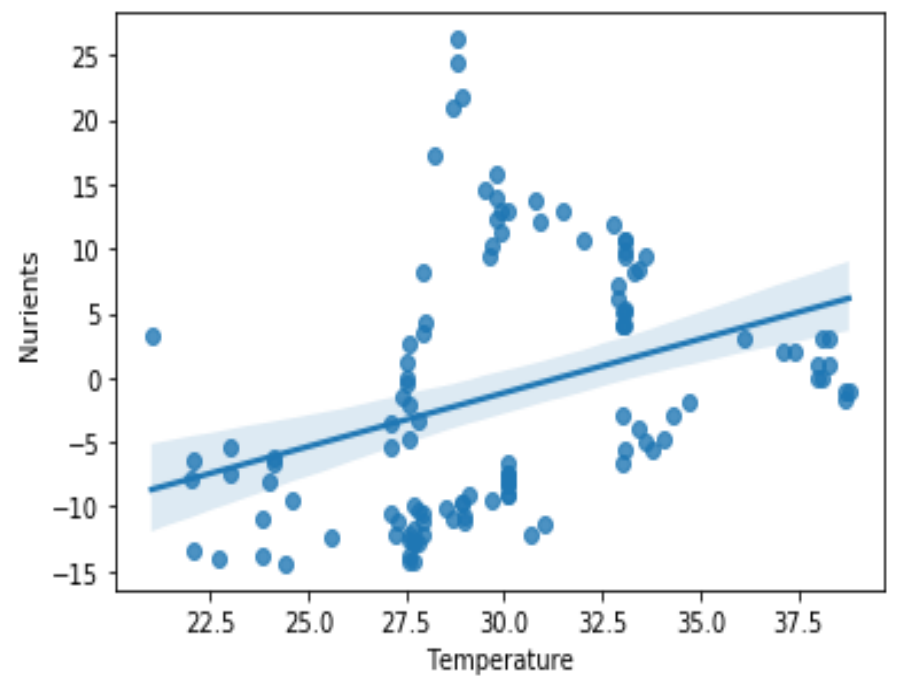

Fig. 4. Temperature Vs Nutrients Requirement

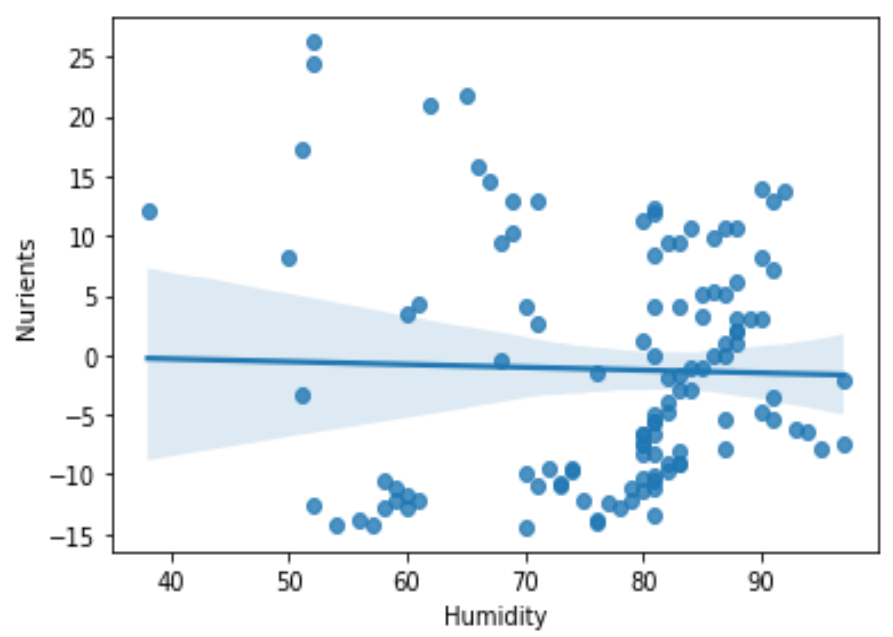

Fig. 5. Humidity (\%) Vs Nutrients Requirement $(\mathrm{mL})$

The red zone is where humidity is higher which enhances the growth rate of the plant, But the green zone is the zone where the plant was sustained. The main system can be running into 2 modes: Low Maintenance Mode and Higher Growth Mode just with the help of climatic condition. 


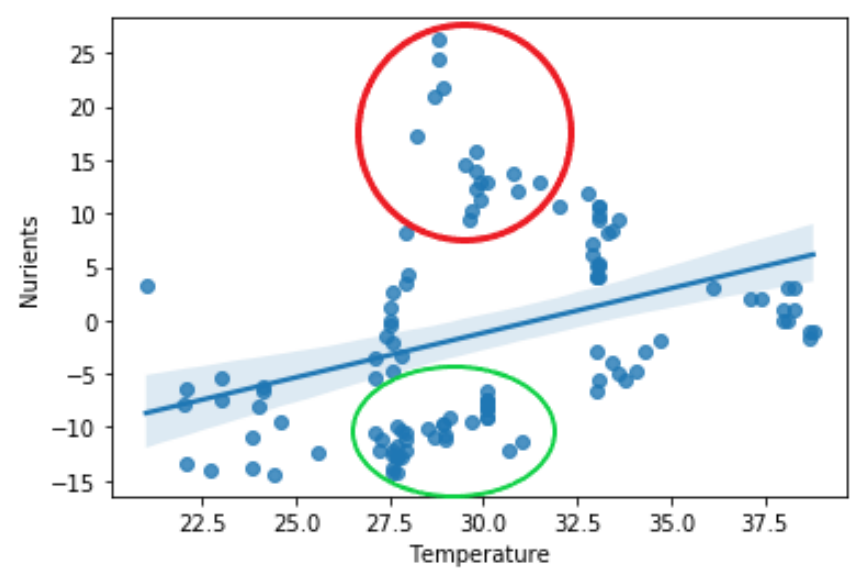

Fig. 6. Temperature Vs Nutrient Requirement with Marked Regions

While training the model including temperature and humidity to predict the requirement of nutrients, we observed an anomaly such that the system never gets stable.
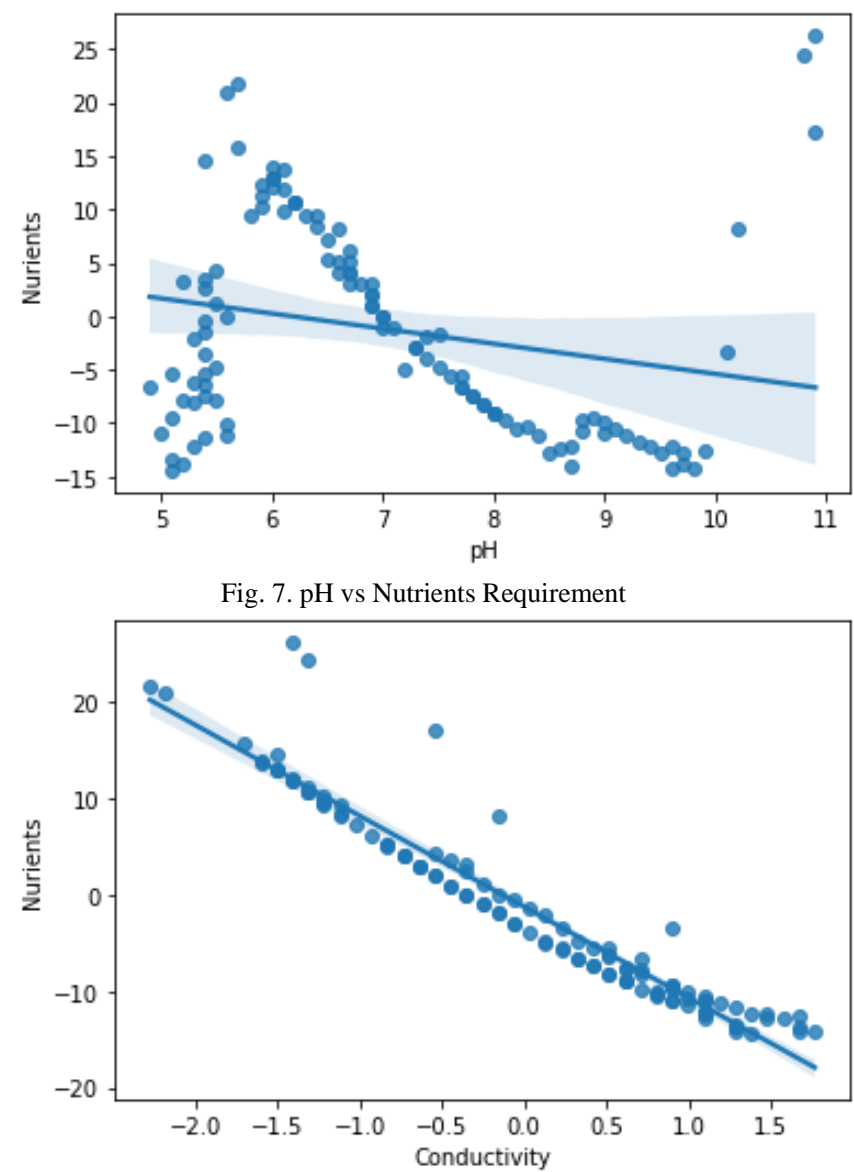

Fig. 8. Conductivity Vs Nutrient requirement

So the model was developed using only $\mathrm{pH}$ level and conductivity. Individually, we got a relation as shown in the graphs. We observe a second-order relation with $\mathrm{pH}$ and first-order relation with conductivity.

But the overall analysis showed an anomaly: the higherorder solutions were showing overfitting even though $\mathrm{pH}$ had a second-order equation [3].

The equation after Polynomial Analysis was: $\mathrm{n}=1.555 \mathrm{p}-10.04 \mathrm{c}-1.1994$
Where $\mathrm{n}$ : Nutrient Requirement in gram per mole water $\mathrm{p}$ : $\mathrm{pH}$ level of water

c: conductivity of water

The graphical representation of predicted values to actual values can be seen.

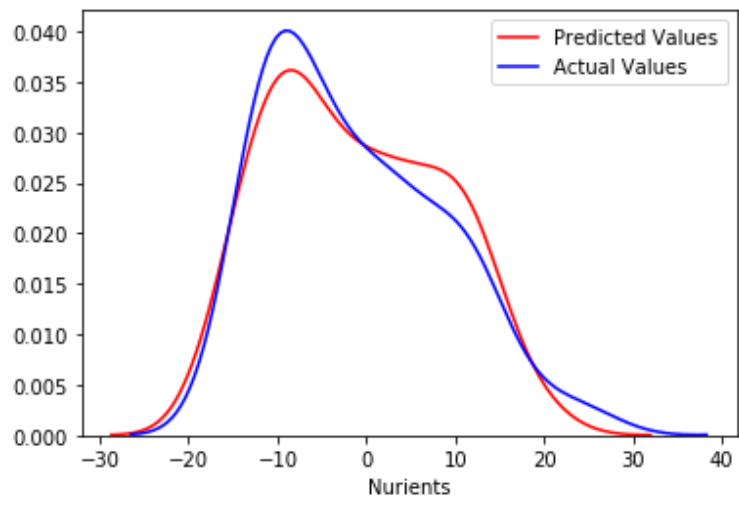

Fig. 9. Prediction of Model and Comparison with Actual Values

\section{V.MOBILE APPLICATION}

To monitor all the system parameters distantly, we developed an Android Application. This application comes with an interactive-secured interface. When we open the application we find a login/signup page.

The page will have a 'User Interface' (Fig. 10. (a)). This login process will ensure proper security for the data in the system. After entering the required credentials, the user will be redirected to the next page of the Application.

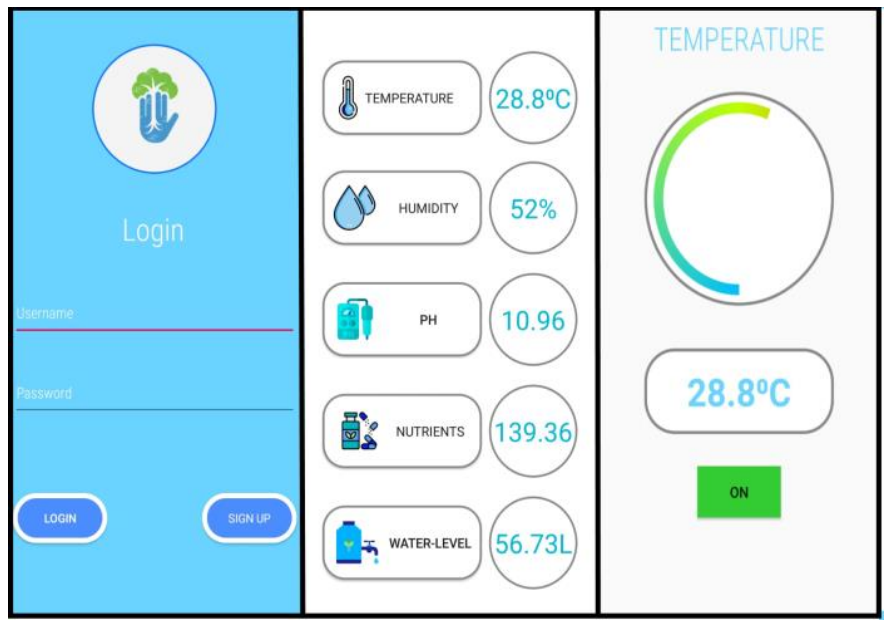

(a)

(b)

(c)

Fig. 10. (a)(b)(c) Screenshots of Application

The next page will consist of menus for different parameters i.e. Temperature, Humidity, PH-level, Nutrients, Water level which we will be monitoring for the plants growing in the system (Fig. 10(b)).

The User Interface of the system is made in such an easier manner that each of the parameters will have a title image alongside, describing it. Each parameter will show the corresponding reading alongside, in a small bubble.

When we click on any one of these quantities, it will redirect us to the next page of the application which will show the corresponding reading in a visual and textual format (Fig. 10 (c)). This figure shows the total description 
of Nutrients of the plants in our system. It will also consist of an On-Off switch for controlling that particular sensor.

\section{VI.SENSORS}

Sensors and actuators along with Microcontroller like Arduino form an integral part of the system to keep a check on various environmental parameters. The Sensors used in the system are:

\section{A. pH sensor and probe:}

A pH sensor is typically used to check the quality of water or any other solution. It measures the amount of alkalinity and acidity in solutions. When used correctly, $\mathrm{pH}$ sensors can also be used to check the quantity of impurities in the solution. In hydroponics, a $\mathrm{pH}$ sensor is used to measure the acidity and alkalinity of solution showing a value between $0-14$.

$\mathrm{pH}$ value and what it indicates: -

- A pH of 7 indicates that the solution is neutral.

- If the $\mathrm{pH}$ is between $0-7$ then the solution is acidic.

- $\mathrm{pH}$ is between $7-14$ then the water is alkaline. In our system, the $\mathrm{pH}$ value of the water will help us to determine the quantity of nutrients present in water.

\section{B. Digital Humidity and Temperature (DHT11)} sensor:

DHT11 is a basic, low-cost digital temperature and humidity sensor. It uses an electrical phenomenon humidity detector and a semiconductor device to measure the surrounding atmosphere and then displays a digital signal on the data pin. In this system, it is used to keep a check on the temperature and humidity in the artificially concocted environment.

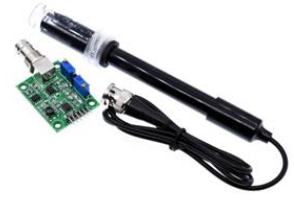

(a)

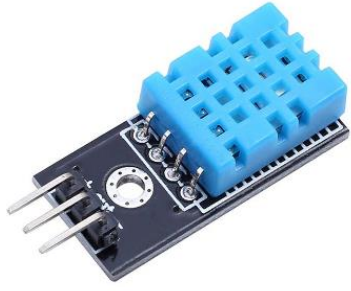

(b)
Fig. 11. (a)pH sensor and Probe, (b)DHT11

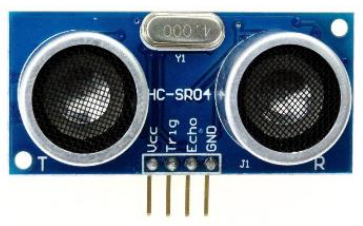

(a)

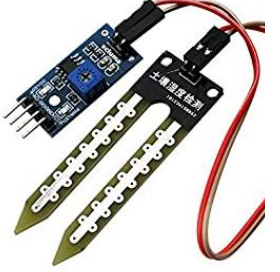

(b)
Fig. 12. (a)HCSR04 Ultrasonic, (b)Soil moisture sensor

\section{Ultrasonic sensor (HCSR-04):}

Ultrasonic sensors convert radio waves to an electric signal and electric signal to audio, hence this sensor is used for non-contact distance measurement. A high-frequency sound wave is generated which is called a signal and it is reflected by the object which is called an echo. The interval between signal and echo is the distance between the object and the ultrasonic sensor. An ultrasonic sensor consists of two membranes. One membrane produces sound, another catch reflected echo. The use of an Ultrasonic sensor in this system to check the level of water in the external tank. The change in water level can be observed with the help of readings.

\section{Soil Moisture sensor:}

Soil Moisture sensors used to measure water content within the soil. Since the direct measurement of free-soil moisture needs removing, drying, and advice of a sample, soil moisture sensors calculate water content indirectly by victimizing another property of the soil, like resistance, nonconductor constant, or interaction with neutrons, as a proxy for the wet content.

\section{VII.OBSERVATIONS AND RESULTS}

The system was kept under control throughout the period of 10 days under specific conditions which was dynamically optimized by equation.

On the first day of setup, the samplings of basil have an average height of $7.6 \mathrm{~cm}$ (measured with scale). The plants were planted in rockwool and were kept in ideal nutrient solution dipped approximately $50 \%$. of rockwool height and were kept in a nursery. The plants were 16 days old on the first day of implementation of the system.

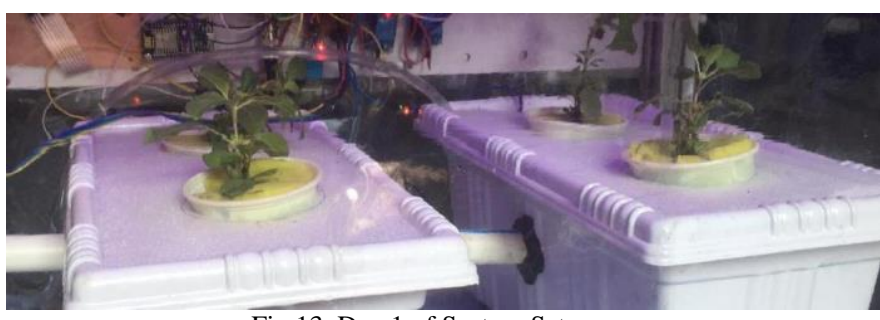

Fig.13. Day 1 of System Setup

Within the period of 10 days of observation we observed that plants grew in height by $57.89 \%$ approximately. The average height of samplings at the end of the system was $12 \mathrm{~cm}$. There was a significant jump in growth within the system as compared to the exterior environment.

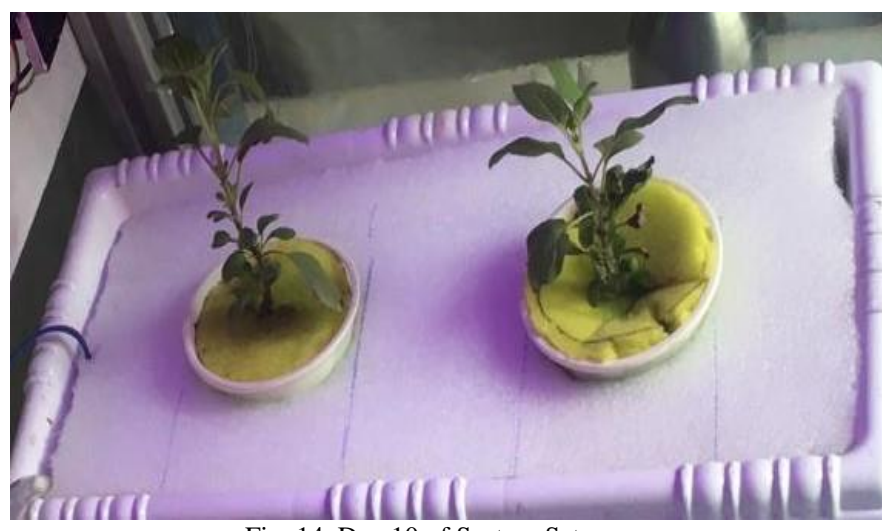

Fig. 14. Day 10 of System Setup 


\section{VIII.CONCLUSION}

This complete work endeavors at automating the hydroponics system along with providing the possibility of distance monitoring and controlling the system using IoT. The hardware of the system along with the sensors, actuators and the software system all work towards obliterating the cumbersomeness of the entire producing process. This system not only breaks through the traditional methods of farming and improves the entire process but also makes it self-sufficient enough to produce anything at any time of the season. The software is integrated to the hardware of the actual system such that it displays the various parameters like Temperature, Humidity, $\mathrm{pH}$ levels etc. on the application that can be downloaded on the smartphones.

Hydroponics helps in faster growth of plants and along with our regulating, monitoring and automated system, described diffusely in this article it obliquely meets the demand of the market. The traditional farming techniques can easily be replaced by these methods and can be implemented on scales desired by the producer depending on the amounts of produce to be expected.

\section{REFERENCES}

[1] Sardare, Mamta. (2013). “A REVIEW ON PLANT WITHOUT SOIL - HYDROPONICS”. International Journal of Research in Engineering and Technology. 02. 299-304. 10.15623/ijret.2013.0203013.

[2] E. Okemwa, "Effectiveness of Aquaponic and Hydroponic Gardening to Traditional Gardening," International Journal of Scientific Research and Innovative Technology, vol. 2, no. 2313-3759, p. 35, 2015.

[3] Fahad, Sk \& Yahya, Abdulsamad. (2018). Big Data Visualization: Allotting by R and Python with GUI Tools. 10.1109/ICSCEE.2018.8538413. 